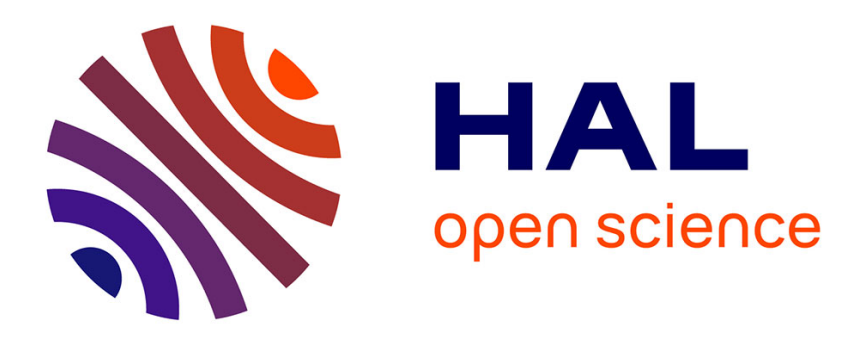

\title{
Pour une conception centrée-utilisateurs des bibliothèques numériques
}

\author{
Jérôme Dinet
}

\section{To cite this version:}

Jérôme Dinet. Pour une conception centrée-utilisateurs des bibliothèques numériques. Communication \& langages, 2009, pp.59-74. halshs-00534026

\section{HAL Id: halshs-00534026 https://shs.hal.science/halshs-00534026}

Submitted on 8 Nov 2010

HAL is a multi-disciplinary open access archive for the deposit and dissemination of scientific research documents, whether they are published or not. The documents may come from teaching and research institutions in France or abroad, or from public or private research centers.
L'archive ouverte pluridisciplinaire HAL, est destinée au dépôt et à la diffusion de documents scientifiques de niveau recherche, publiés ou non, émanant des établissements d'enseignement et de recherche français ou étrangers, des laboratoires publics ou privés. 


\section{Pour une conception centrée-utilisateurs des bibliothèques} numériques

\section{Contexte et enjeux}

Dans cet article, nous nous intéressons aux comportements réels des usagers des bibliothèques numériques, à leurs attentes et à leurs perceptions en défendant l'idée suivante : l'usager et ses caractéristiques doivent être pris en compte dès la conception d'une bibliothèque numérique ${ }^{1}$. En ce sens, nous défendons une conception centrée-utilisateur en opposition avec l'approche techno-centrée trop souvent constatée.

Lorsque sont interrogés les concepteurs, commanditaires et professionnels en charge des bibliothèques numériques, les principaux inconvénients évoqués par certains concernent les dimensions techniques ou réglementaires telles que l'obsolescence technologique, le coût lié à la maintenance et à l'actualisation des contenus, le problème des droits d'auteurs et d'accès, l'interopérabilité entre les systèmes, l'augmentation des flux d'information. Les usagers et leurs éventuelles difficultés à utiliser les bibliothèques numériques sont trop rarement abordés. S'il est vrai que certains verrous technologiques et juridiques perdurent, ce sont essentiellement les "verrous humains » qui expliquent en grande partie les non-usages, les difficultés et les abandons.

L'ergonomie permet de lever certains de ces verrous, à l'aide d'apports conceptuels et méthodologiques

1. Dinet, J., 2008, Usages, usagers et compétences informationnelles au XXI $I^{e}$ siècle, Hermes, Paris.
LES BIBLIOTHËQUES NUMÉRIQUES

\section{JÉRÔME DINET}

Pour une conception centrée-utilisateurs des bibliothèques numériques

Cet article s'intéresse aux comportements réels des usagers qui interagissent avec des bibliothèques numériques (Digital Libraries, ou DL) et analyse leurs principales difficultés. Dans cette perspective, il analyse et discute les méthodes traditionnellement utilisées pour étudier les besoins réels et les motivations des utilisateurs finaux en défendant le point de vue suivant : nous devons absolument étudier les besoins réels et les véritables comportements des utilisateurs en combinant des études empiriques à des études expérimentales. Si au début des études sur les comportements des utilisateurs, la phrase "Si nous construisons, ils viendront" était le leitmotiv des chercheurs et développeurs des DL, il est aujourd'hui nécessaire de mieux comprendre les besoins et les comportements des usagers si nous voulons créer des bibliothèques pertinentes, utiles et utilisables pour ces mêmes utilisateurs.

Mots-clés : Bibliothèques numériques, Besoins, Usager, Psychologie, Interaction 
innovants qui empruntent à plusieurs autres disciplines (psychologie, sciences de l'ingénieur, informatique, etc.). L'une des problématiques de l'ergonomie est l'adaptation des outils et systèmes au fonctionnement cognitif des utilisateurs : c'est l'ergonomie cognitive. L'ergonomie logicielle se situe du côté de l'ergonomie cognitive. Son objectif est d'une part, d'améliorer l'interaction homme ordinateur et d'autre part, de faire en sorte que toute application informatique soit utile (répondre aux besoins des utilisateurs pour lesquels il a été conçu) et utilisable (ou maniable). Dire qu'un système tel une bibliothèque numérique est " ergonomique » ne veut rien dire en soi : un système est ergonomique pour une personne donnée ayant un objectif donné, à un moment donné dans un contexte social précis. De plus, l'ergonomie répond à un certain nombre de critères clairement identifiés dont l'appréciation doit obéir à des règles scientifiques et rigoureuses $^{2}$.

Dans cet article, nous partons du double constat suivant : d'une part, les attentes des commanditaires, des concepteurs et des usagers divergent souvent; d'autre part, les comportements réels et opinions des usagers sont rarement pris en compte et lorsqu'ils le sont, c'est tardivement. Or, les sommes allouées au développement de nouvelles bibliothèques numériques et/ou à la maintenance de celles existant déjà semblent énormes. "Semblent » car il est extrêmement difficile de connaître les coûts directs et indirects liés aux bibliothèques numériques. Quelques rares études permettent néanmoins de prendre conscience des enjeux économiques. À titre d'exemple, pour une seule année, le Royaume-Uni a injecté plus de 25 millions de Livres sterling ${ }^{3}$ (environ 33 millions d'euros) pour créer et maintenir les bibliothèques numériques. Or, comme le souligne un nombre croissant de spécialistes, les usages et bénéfices réels des bibliothèques numériques sont encore méconnus. Certains auteurs s'intéressent particulièrement aux divergences de points de vue entre les commanditaires et les concepteurs des environnements numériques ${ }^{4}$ en montrant, à l'aide d'études empiriques, que la collaboration en amont permet d'éviter certains écueils ergonomiques qu'il aurait été facile de dépister et de corriger.

\section{"If we build it, they will come "}

Tel semble être le leitmotiv de nombreux concepteurs de bibliothèques numériques et de décideurs politiques : "si nous construisons, ils viendront ». Le pronom « ils » correspond aux usagers tandis que le « nous » correspond aux concepteurs ${ }^{5}$. Dans l'un de ses derniers articles qui ressemblent à une profession de foi d'un individu se sentant investi d'une mission quasi-divine, P. Michel ajoute que le rôle

2. Pour une synthèse : Brangier, E., \& Barcenilla, J., 2003, Concevoir un produit facile à utiliser : adapter les technologies à l'homme, Editions d'Organisation, Paris.

3. Creaser, C., Hamblin, Y., \& Davies, J.E., 2006, « An assessment of potential efficiency gains through online content use ». Program: Electronic library and information systems, 40 (2), pp. 178-189.

4. Chevalier, A., 2005, «Evaluer un site Web : les concepteurs et les utilisateurs parviennent-ils à identifier les problèmes d'utilisabilité ? " Revue d'Intelligence Artificielle, 19, pp. 319-338 ; Chevalier, A., \& Kicka, M., 2006, «Web Designers and Web Users: Influence of the Ergonomic Quality of the Web Site on the Information Search ", International Journal of Human-Computer Studies, 64 (10), pp. 1031-1048.

5. Michel, P., 2005, «Digitizing special collections : to boldly go where we have been before », Library Hi Tech, 23 (3), pp. 379-395, traduction par Jérôme Dinet. 
des concepteurs et décideurs est de construire des bibliothèques numériques telles des étoiles guidant les usagers vers de nouveaux horizons qu'ils ne soupçonnent même pas.

Certains concepteurs et décideurs semblent plus réservés quant à leur mission et semblent plus lucides quant aux usages réels qui sont faits des environnements qu'ils conçoivent. Ainsi, Wilson ${ }^{6}$ définit la mission des concepteurs des bibliothèques numériques de la manière suivante : " nous construisons, dans l'espoir que quelqu'un viendra, sans savoir ce que nous construisons exactement ni savoir qui viendra » (notre traduction).

Enfin, quelques concepteurs de bibliothèques numériques n'hésitent pas à parler de gaspillage d'une part, de l'effort intellectuel et d'autre part, de l'argent public lorsqu'ils constatent avec désarroi que la plupart des environnements numériques n'est pas utilisée ou l'est mal'. Certains auteurs avaient déjà souligné ce gaspillage il y a plusieurs années ${ }^{8}$ sans avoir été entendus semble-t-il. Jones, Gay et Rieger insistaient également sur l'importance à accorder aux besoins réels des usagers et sur la nécessité de mener des études sérieuses avant d'entreprendre la constitution des vastes bibliothèques numériques.

Ainsi, si certains continuent à penser que «l'offre conditionnera les usages », la réalité est tout autre lorsque l'on regarde de plus près les usages. Dans une étude récente, $76 \%$ des usagers auxquels il est demandé de rechercher des références d'ouvrages dans une bibliothèque numérique " grand public » ne parviennent pas à réaliser ces tâches pourtant simples 9 . De plus, bien que confrontés à un problème, plus de $80 \%$ des usagers ayant participé à l'étude n'ont pas utilisé l'aide disponible : le principal argument avancé par ces usagers est que les fonctions d'aide sont généralement destinées à des experts (avec un vocabulaire complexe et trop technique) et ne sont pas assez intuitives. Quoi qu'il en soit, les performances des usagers apparaissent souvent "décevantes » aux yeux des concepteurs et commanditaires des bibliothèques numériques. L'un des obstacles à la prise en compte de ces faibles performances des usagers est l'existence de fausses croyances $^{10}$.

\section{LES FAUSSES CROYANCES}

Il existe des croyances, que nous qualifions de fausses car erronées, chez les concepteurs et/ou les usagers. Ces croyances concernent les nouvelles technologies

6. Wilson, L.A., 2003, " If we build it, will they come? Library users in a digital world ». In S.H. Lee (Ed.), Improved access to information : portals, content selection, and digital information, Haworth Information Press, Binghamton, New York, pp. 19-28.

7. Warwick, C., Terras, M., Huntington, P., \& Pappa, N., 2006a, « If you build it will they come? The Lairah Study: Quantifying the Use of Online Resources in the Arts and Humanities Through Statistical Analysis of User Log Data », in The Proceedings of digital humanities, pp. 225-228.

8. Jones, M.L.W, Gay, G.K, \& Rieger, R.H., 1999, « Comparing Evaluations of Digital Collection Efforts ", DLib Magazine, 5 (11), accessible en ligne : http://www.dlib.org/dlib/november99/11jones.html. Consulté le 10 juillet 2008.

9. Kani-Zabihi, E., Ghinea, G., \& Chen, S.Y., in press, «User perceptions of online public library catalogues ", International Journal of Information Management.

10. Hansen, P., \& Jarvelin, K., 2005, « Collaborative Information Retrieval in an information-intensive domain », Information Processing \& Management, 41 (5), pp. 1101-1119. 
et en particulier Internet et sont, pour certaines, largement entretenues par le discours médiatique ambiant.

Fausse croyance $\mathrm{n}^{\circ} 1$ : « les usagers manipulent et maîtrisent de mieux en mieux les nouvelles technologies ». Il ne faut pas confondre l'aisance avec laquelle les utilisateurs manipulent les périphériques et/ou certains objets techniques (tels que les télécommandes ou les joysticks) avec l'aisance dans la manipulation des flux d'information qui sont véhiculés par ces périphériques ou objets techniques. Nous pouvons ainsi distinguer deux types de connaissances procédurales qui sont nécessaires à acquérir et développer : les connaissances procédurales liées à la manipulation des périphériques et les connaissances procédurales liées à la démarche. Or, le risque est grand de croire que les usagers " comprennent tout ce qui se passe derrière l'écran » parce qu'ils manipulent sans crainte le clavier et la souris et qu'ils n'hésitent pas à se "lancer sur la toile ». De plus, deux remarques sont importantes à noter : d'une part, tous les usagers des bibliothèques numériques n'aiment pas les ordinateurs; d'autre part, d'un point de vue purement manipulatoire, l'utilisation des ordinateurs peut se révéler difficile pour certains. Il a longtemps été posé comme acquis que les difficultés dans la manipulation des nouvelles technologies que rencontraient certains usagers allaient disparaître avec la fréquence d'utilisation et donc, l'expérience au sens de confrontation avec les nouvelles technologies. Si cette opinion est vraie en ce qui concerne la majorité des jeunes utilisateurs, elle est à modérer. Dans une étude dans laquelle des élèves de trois classes d'âge différentes devaient soit pointer des objets sur l'écran avec la souris (pointing) soit déplacer des objets avec cette même souris (dragging), Joiner et al. ${ }^{11}$ ont montré que, non seulement les jeunes utilisateurs (de 5 à 9 ans) mettaient beaucoup de temps à réaliser ces deux activités, mais que leurs performances étaient relativement faibles. En d'autres termes, la maîtrise de la souris pose des difficultés à certains des plus jeunes utilisateurs. Cette constatation a également été relevée dans des études postérieures ${ }^{12}$. Ces mêmes auteurs ont également relevé que l'utilisation du clavier alphanumérique pose quelques problèmes à certains élèves. Des résultats identiques ont récemment été trouvés chez des usagers plus âgés ${ }^{13}$ pour lesquels la manipulation des périphériques n'est pas si évidente que cela.

Fausse croyance $\mathrm{n}^{\circ} 2$ : «tous les jeunes aiment les ordinateurs et Internet». Certains jeunes utilisateurs présentent une défiance, parfois une crainte voire une anxiété vis-à-vis des ordinateurs. En effet, il est faux de croire que parce qu'un usager est jeune, il aime les ordinateurs ! D’ailleurs, depuis quelques années, un

11. Joiner, R., Messer, D., Light, P., \& Littleton, K., 1998, « It is best to point for young children: A comparison of children's pointing and dragging ", Computers in Human Behavior, 14 (3), pp. 513-529.

12. Borgman, C.L., Hirsh, S.G., Walter, V.A., \& Gallagher, A.L., 1999, « Children's searching behavior on browsing and keyword online catalogs : The science library catalog project ", Journal of the American Society for Information Science, 46 (9), pp. 663-684 ; Dinet, J., \& Tricot, A., 2008, " Recherche d'information dans les documents électroniques ", in A. Chevalier \& A. Tricot (Eds.), Ergonomie cognitive des documents électroniques, PUF, Paris, pp. 35-69.

13. Chevalier, A., Dommes, A., \& Marquié, J.-C., 2008, « Les Seniors et les Technologies de l'Information et de la Communication : le cas d'Internet ", in J. Dinet (Ed.), Usages, usagers et compétences informationnelles au XXI $I^{e}$ siècle, Hermes, Paris. 
nombre croissant d'études s'intéresse à cette anxiété liée à l'ordinateur, cette anxiété pouvant perdurer chez des adultes refusant catégoriquement d'utiliser les nouvelles technologies ${ }^{14}$. Longtemps, il a été suggéré que l'anxiété liée à l'ordinateur (computer anxiety), pouvant parfois déboucher sur des cas de véritables phobies (computerphobia), allait décroître jusqu'à disparaître au fil des années puisque nous serions de plus en plus confrontés, dans tous nos environnements, aux outils technologiques. Or, dans une vaste étude conduite de 1992 à 1998, dans laquelle ont été suivis et interrogés 1051 étudiants inscrits en première année universitaire, Todman ${ }^{15}$ a montré que, même si cette anxiété décroissait globalement, elle demeurait présente, surtout chez les femmes. Pourtant, rappelons que les participants à cette étude étaient tous des étudiants de première année universitaire, c'est-à-dire étaient tous des individus ayant suivi un cursus scolaire des premier et second degrés «traditionnels » et ayant fréquemment utilisé les ordinateurs et l'Internet.

Fausse croyance $\mathrm{n}^{\circ} 3$ : «quantité = qualité d'information ». Lorsqu'il est demandé aux usagers d'effectuer des recherches d'informations sur Internet ou dans tout autre environnement documentaire numérique, il n'est pas rare de voir revenir certains avec des " paquets » d'informations ou des listes d'impressions de pages Internet et/ou de références documentaires. Ces usagers (élèves ou étudiants) semblent être dans une stratégie quantitative au détriment d'une stratégie qualitative, cette dernière nécessitant de lire, comprendre et évaluer la pertinence des informations ou références retenues.

Fausse croyance $\mathrm{n}^{\circ} 4$ : « je peux me passer de l'expert et être autonome ». La facilité d'accès aux ordinateurs et à leur contenu laisse croire à certains usagers que l'expert (l'enseignant, le documentaliste, le bibliothécaire, etc.) ne leur est d'aucune utilité. Au niveau du collège et du lycée, certains professeurs documentalistes relatent même des cas d'élèves qui pénètrent dans le CDI, travaillent quelque temps sur un ordinateur et ressortent d'une part, sans avoir trouvé l'information recherchée et d'autre part, sans leur avoir demandé de l'aide et/ou un conseil. Lors de certaines observations de recherches documentaires menées dans des BCD d'écoles primaires, il nous a été donné de voir des élèves sortir ainsi bredouilles et annoncer qu' «il n'y a rien à la bibliothèque » car le logiciel leur a proposé « 0 référence » à leur requête. Or, après examen de ces requêtes, il s'est avéré que plusieurs contenaient simplement des erreurs orthographiques faisant obstacle à une recherche pertinente par le logiciel.

Fausse croyance $n^{\circ} 5$ : « outil = démarche ». La recherche d'information et/ou documentaire est avant tout une démarche intellectuelle. Or, pour certains usagers, il y a une confusion entre la démarche de recherche d'information et les outils qu'il est possible d'utiliser pour effectuer une recherche d'information. Ainsi, lorsque nous demandons à des jeunes usagers (collégiens, lycéens ou étudiants) s'ils savent rechercher des informations sur Internet, beaucoup répondent « oui,

14. Pour une synthèse : Thorpe, S.J., \& Brosnan, M.J., 2007, « Does computer anxiety reach levels which conform to DSM IV criteria for specific phobia? ", Computers in Human behavior, 23, pp. 1258-1272.

15. Todman, J., 2000, "Gender differences in computer anxiety among university entrants since 1992 », Computers \& Education, 34 (1), pp. 27-35. 
car je sais utiliser Google ». Or, plusieurs étapes liées à la recherche d'information et/ou documentaire (par exemple, le choix des mots-clés, le choix des sources d'informations) peuvent se réaliser indépendamment de la présence d'outils informatiques.

Si être conscient que ces croyances sont erronées est une nécessité, ce n'est pas suffisant pour que soient correctement appréhendés les comportements réels des usagers. Leurs attentes et véritables besoins doivent être identifiés avant la conception des bibliothèques numériques.

\section{LES ATTENTES DES USAGERS}

Une étude récente a déterminé, â l'aide d'entretiens et de questionnaires conduits auprès d'usagers de différents niveaux d'expertise (novice/intermédiaire/expert) devant accomplir des recherches documentaires dans quatre bibliothèques numériques différentes ${ }^{16}$, il a été montré que les trois principales attentes des utilisateurs des bibliothèques numériques sont les suivantes :

- les contenus doivent être facilement accessibles et ne pas exiger de compétences techniques ou de connaissances en documentation particulières ;

- une bibliothèque numérique doit offrir les mêmes services qu'une bibliothèque traditionnelle tels que l'affichage des dernières nouveautés acquises, la possibilité de gérer les emprunts des ouvrages, etc. ;

- une bibliothèque numérique doit être conçue de manière à ce que l'utilisateur retrouve ses " marques » telles que l'obtention de l'aide d'employés.

Il est à noter que ces trois attentes sont rarement comblées par les bibliothèques numériques actuelles. L'analyse plus précise des entretiens a permis à Kani-Zabihi et al. de distinguer des attentes couvrant trois domaines distincts mais complémentaires :

\section{Au niveau des fonctionnalités}

- les résultats proposés par le moteur de recherche interne à la bibliothèque numérique devraient pouvoir être filtrés et/ou organisés et/ou classés par l'usager ;

- les contenus, ou du moins une partie des contenus, devraient être téléchargeables;

- un historique personnel devrait toujours être disponible afin que chaque usager puisse stocker ses recherches et les résultats liés et ainsi les retrouver lors de ses futures utilisations ;

- les systèmes devraient permettre et supporter la recherche collaborative d'information (par exemple, par le partage des « paniers » entre plusieurs utilisateurs). 


\section{Au niveau de l'interface et de l'utilisable}

- le format de présentation des résultats (références et/ou documents) devrait être identique et homogène ;

- les icônes et boutons devraient être "parlants » à un usager tout-venant et ne pas emprunter à un vocabulaire spécialisé et/ou trop vague : ainsi, 35,1\% des usagers interrogés ne comprennent pas exactement à quoi sert le "panier " présent sur l'interface d'une bibliothèque numérique (puisque le "panier " est généralement rencontré sur les sites commerciaux) et ignorent sa durée de rétention;

- les résultats d'une recherche devraient tenir sur une seule page ;

- des filtres et recherches multicritères devraient non seulement être permis pour classer et organiser les résultats mais également être facilement visibles: dans cette même étude, dans l'une des tâches qui leur étaient demandées, $90 \%$ des usagers n'ont pas vu (et n'ont donc pas utilisé) le bouton permettant de n'obtenir que des DVD;

\section{Au niveau du contenu}

- une bibliothèque numérique devrait contenir tous les formats (vidéos, images, sons, etc.) ;

- une bibliothèque numérique devrait permettre de passer à d'autres bibliothèques numériques aisément.

Même si certains des souhaits exprimés sont irréalisables à cause de difficultés techniques ou peu pertinents pour des professionnels de la documentation, l'intérêt d'une telle étude est de s'intéresser aux besoins des usagers.

D'autres attentes plus spécifiques ont été retrouvées dans des études similaires. Par exemple, la présence, même virtuelle, d'un documentaliste ou d'un expert du domaine a été exprimée par les usagers interrogés par Inskip et al. ${ }^{17}$. Ces auteurs se sont intéressés aux attentes d'usagers d'une bibliothèque numérique spécialisée dans un domaine très particulier : la musique folk (la Vaughan Williams Memorial Library : http://www.efdss.org/library.htm). Les usagers interrogés étaient de différents niveaux : des professionnels du monde de la musique folk (journalistes spécialisés, historiens de la musique, etc.), des enseignants et étudiants, des performeurs (musiciens, danseurs, acteurs, etc.), des passionnés. Le principal regret des usagers interrogés a effectivement été l'absence de contact avec des professionnels et experts du même domaine. Plusieurs ont aussi regretté l'absence d'un forum pour partager les opinions et avis entre usagers de cette bibliothèque numérique. A alors été avancée, par les usagers eux-mêmes, l'idée d'un documentaliste numérique (digital librarian) qui pourrait assurer ce contact. En effet, les documentalistes sont une (res)source d'information essentielle pour les usagers. Il est donc essentiel que les services Web conservent ces documentalistes et leur fassent une place. Plusieurs pistes sont avancées par Inskip et al. : la possibilité

17. Inskip, C., Butterworth, R., \& MacFarlane, A., 2008, «A study of the information needs of the users of a folk music library and the implications for the design of a digital library system ", Information processing and Management, 44, pp. 647-662. 
de contacter un documentaliste par courrier électronique ; la création de blogs associés aux bibliothèques numériques; la possibilité de créer et gérer des foires aux questions (FAQ). Mais, ces auteurs insistent sur le fait que pour les domaines très spécifiques (tels que la musique folk), la majorité des usagers interrogés ont insisté sur l'importance du contact humain pour l'échange des impressions, des goûts, des émotions. En effet, le contact avec de "vrais » interlocuteurs semble primordial dans le cas de ces bibliothèques numériques liées aux arts (musique, littérature, peinture, etc.), où l'affect et les émotions sont prépondérants. Des résultats similaires avaient déjà été trouvés antérieurement ${ }^{18}$.

L'accès rapide à l'information pertinente est un enjeu crucial. Certains ont même rapproché ce goût accru pour la vitesse d'accès aux informations en l'assimilant à la génération «zapping ». Pourtant, comme le fait remarquer $\operatorname{Tricot}^{19}$, il vaut mieux parler de traitement superficiel des informations que de zapping. En effet, chez les jeunes usagers notamment, de nombreuses études ont montré que ces derniers se contentaient souvent des premières pages (pour ne pas dire de la première page) pour évaluer si le document et/ou le site trouvé étaient pertinents. Même si l'on peut regretter l'absence d'un traitement (au sens de lecture-compréhension) plus minutieux et plus profond des informations de la part des jeunes usagers, le fait est là. De même, des études récentes tendent à montrer que l'ordre des résultats d'une recherche documentaire est un facteur déterminant pour les jeunes lecteurs puisqu'ils ne traitent généralement que les premières références proposées par le système. Mais, il serait faux de penser que cette volonté d'avoir rapidement les informations recherchées ne concerne que les jeunes usagers.

L'accès aux résumés, condensés des informations contenues dans un article ou document, semble être primordial pour les usagers, y compris les experts. Dans une étude récente, Nicholas et al. ${ }^{20}$ se sont intéressés à la nature des informations auxquelles les utilisateurs de la base de données ScienceDirect accédaient réellement. Ils ont utilisé la technique de l'analyse des fichiers de transaction logs (véritables traces informatiques de l'activité de l'usager) pour connaître précisément les comportements de navigation des lecteurs-chercheurs d'informations. Les participants à cette étude étaient tous des chercheurs et/ou des enseignants-chercheurs de diverses disciplines, c'est-à-dire des experts dans leur domaine. L'étude a duré 18 mois et a ainsi permis de " tracer » avec précision les comportements réels de ces utilisateurs. Sur la base des données recueillies, cinq stratégies possibles pour l'usager confronté à la liste des articles proposés après avoir interrogé la base de données Sciencedirect ont été distinguées : 1. lire uniquement le résumé ; 2 . lire l'article complet en version html uniquement ; 3. lire l'article complet en version pdf uniquement ; 4 . lire le résumé avant de lire la

18. Moyo, L.M., 2004, "Electronic libraries and the emergence of new service paradigms ", The Electronic Library, 22 (3), pp. 220-230.

19. Tricot, A, 2000, "Je zappe, j'apprends ? ", Savoir-CDI, revue en ligne : http://savoirscdi. cndp.fr/archives/dossier_mois/tricot/tricot.htm

20. Nicholas, D., Huntington, P., \& Jamali, H.R., 2007, " The use, users, and role of abstract in the digital scholarly environment », The Journal of Academic Librarianship, 33 (4), pp. 446-453. 
version html ; 5. lire la version html avant de lire la version pdf. L'un des résultats les plus surprenants est que, contrairement à ce que déclarent habituellement les chercheurs et enseignants-chercheurs, $20 \%$ d'entre eux se contentent de lire le résumé des articles (stratégie numéro 1). Une analyse plus fine montre que ces résultats diffèrent selon la discipline universitaire du lecteur : ainsi, $41 \%$ des chercheurs en sciences humaines et sociales ne lisent que les résumés, ce qui ne les empêche pas par la suite de citer les études correspondant aux résumés dans leurs enseignements et/ou propres publications (études qu'ils n'ont donc pas lues dans leur totalité).

Ce même article s'est également intéressé à d'autres bases de données hyperspécialisées et destinées à des experts (telles que Nucleic Acids Research ou NAR). Les résultats convergent tous dans le même sens : les résumés sont une source d'informations primordiale pour les usagers et parfois même, la seule source d'informations. Encore une fois, notre propos n'est pas de dénoncer telle ou telle pratique, mais de décrire sur la base d'études scientifiques des faits réels, d'autant plus que certains concepteurs commencent à porter un intérêt aux clients-usagers, leurs comportements et leurs attitudes.

\section{DE LA DifFusion d'UNE INNOVATION À SON ACCEPTATION}

Les commanditaires, les organismes payeurs et même les concepteurs des bibliothèques numériques s'interrogent souvent sur les raisons qui expliquent qu'un système ne soit pas utilisé. Si nous considérons la bibliothèque numérique comme une innovation, alors la théorie de la diffusion de l'innovation élaborée par Rogers $^{21}$ peut permettre de comprendre et d'identifier certains freins à l'acceptation d'une bibliothèque numérique. Est considérée comme une innovation « toute idée, pratique ou objet perçus comme nouveau par un individu ou un groupe social» (traduction de Dinet, 2008). En d'autres termes, un système n'est pas intrinsèquement innovant : c'est le regard de l'usager (ou futur usager) qui confère à ce système le caractère innovant.

La théorie de la diffusion de l'innovation initialement proposée en 1962 par Everett Rogers a été appliquée autant sur le plan individuel ${ }^{22}$ que sur le plan organisationnel ${ }^{23}$. Bien que ne concernant pas uniquement les technologies informatiques, elle offre un cadre conceptuel au concept d'acceptabilité car son but est d'expliquer comment une innovation technologique évolue du stade d'invention à celui d'utilisation élargie. Selon Rogers, il existerait cinq éléments qui détermineraient l'adoption ou la diffusion d'une nouvelle technologie :

- l'avantage relatif (relative advantage) est le degré auquel une innovation est perçue comme étant meilleure que celles qui existent déjà. Il n'est pas nécessaire que cette innovation possède effectivement plus d'avantages que les autres : ce qui est important est que l'individu la perçoive comme étant avantageuse ;

21. Rogers, E., 1995, Diffusion of innovation, Free Press, New-York, $4^{\text {th }}$ edition.

22. Rogers, 1995, op. cit.

23. Zaltman, G., Duncan, R., \& Holbeck, J., 1973, Innovation and organizations, John Wiley, New-York. 
- la compatibilité (compatibility) est une mesure du degré auquel une innovation est perçue comme étant consistante avec les valeurs socioculturelles existantes, les expériences passées, les pratiques sociales et les normes des utilisateurs. Une idée qui serait incompatible avec les valeurs et normes actuelles prendrait plus de temps à être adoptée qu'une innovation compatible. De même, dans certains cas, l'adoption d'une innovation compatible nécessite l'adoption au préalable d'un nouveau système de valeur, ce qui peut prendre un temps considérable;

- la complexité (complexity) est une mesure du degré auquel une innovation est perçue comme étant difficile à comprendre et à utiliser. Les nouvelles idées qui sont simples à comprendre vont être adoptées beaucoup plus rapidement que d'autres qui nécessitent de développer de nouvelles compétences avant de pouvoir les comprendre ;

- la testabilité (trialability) consiste en la possibilité de tester une innovation et de la modifier avant de s'engager à l'utiliser. L'opportunité de tester une innovation va permettre aux éventuels utilisateurs d'avoir plus de confiance dans le produit car ils auront eu la possibilité d'apprendre à l'utiliser ;

- l'observabilité (observability) est le degré auquel les résultats et bénéfices d'une innovation sont clairs. Plus les résultats de l'adoption de l'innovation seront clairs et plus les individus l'adopteront facilement.

Un examen récent et minutieux de la littérature scientifique a permis d'affiner ${ }^{24}$ chacun de ces cinq éléments en se centrant en particulier sur les environnements numériques liés à l'information. Ainsi, chacun des cinq éléments cités précédemment comporte plusieurs sous-composants qui déterminent l'adoption d'une innovation dans le domaine des nouvelles technologies liées à l'information et à la documentation (tableau ci-dessous).

Les auteurs remarquent toutefois certains décalages entre ce que déclarent les usagers et la réalité. Ainsi, si une aide contextuelle est souvent citée comme précieuse par les usagers, l'examen des comportements réels démontre que de telles aides sont rarement utilisées bien que disponibles.

Quoi qu'il en soit, les concepteurs et commanditaires doivent être pleinement conscients de certaines limites, dont les suivantes :

- l'existence de différentes stratégies de recherche pour un même système : en effet, il est faux de croire qu'à un outil ou dispositif, correspond une et une seule stratégie. S'il est possible de décrire les stratégies les plus optimales et donc de suggérer des manières de "bien faire " aux usagers, de nombreuses stratégies existent selon le niveau d'expérience de l'utilisateur, ses connaissances du domaine, ses compétences langagières, le contexte, etc.;

- la faible confiance qu'ont certains usagers à l'égard des informations retrouvées depuis Internet et les bases de données numériques;

24. Hayati, Z., \& Jowkar, T., 2008, « Adoption of electronic reference materials in academic libraries of Iran », The International Information \& Library Review, 40, pp. 52-63. 


\section{Tableau 1.}

\begin{tabular}{|ll|}
\hline L'avantage relatif & $\begin{array}{l}\text { La possibilité d'utiliser les opérateurs booléens } \\
\text { La possibilité de faire des recherches dans des champs spécifiques } \\
\text { (noms d'auteurs, mots-clés, etc.) } \\
\text { L'existence de liens vers les fichiers d'autorités } \\
\text { La simplicité d'utilisation } \\
\text { La possibilité d'interroger de vastes corpus } \\
\text { La possibilité de conserver et de consulter l'historique de recherche } \\
\text { L'existence de liens hypertextes vers les informations primaires et } \\
\text { documents } \\
\text { L'existence d'un support off-line (CD-Rom) permettant de consulter les } \\
\text { bases }\end{array}$ \\
\hline $\begin{array}{l}\text { L'observabilité } \\
\text { Le délai de réponse du système (feedback) } \\
\text { Le nombre de résultats proposés par le système } \\
\text { L'accès au plein-texte } \\
\text { L'accès à des bases distantes géographiquement } \\
\text { La possibilité d'imprimer les résultats } \\
\text { La possibilité d'interroger plusieurs bases simultanément }\end{array}$ \\
\hline $\begin{array}{l}\text { La complexité, la } \\
\text { testabilité, la } \\
\text { compatibilité }\end{array}$ & $\begin{array}{l}\text { Le coût raisonnable des services } \\
\text { La simplicité et convivialité de l'interface } \\
\text { L'existence d'une aide contextuelle } \\
\text { La possibilité de tester sur des cas fictifs le système }\end{array}$ \\
\hline
\end{tabular}

- les difficultés pour de nombreux usagers tout-venant (i.e., non-experts) pour réaliser des recherches d'informations, même simples;

- le faible niveau des compétences chez certains usagers dans l'utilisation des nouvelles technologies.

Chacune des cinq caractéristiques énoncées ci-dessous prise seule n'est pas suffisante pour prédire l'adoption d'une innovation. Mais des études ont démontré qu'une combinaison de ces caractéristiques (des avantages, une compatibilité avec les croyances et les normes, un niveau de complexité bas, une possibilité de tester l'innovation et un fort degré d'observabilité) conduira à une plus grande probabilité d'adoption de l'innovation ${ }^{25}$. Par ailleurs, une méta-analyse de la littérature portant sur la théorie de la diffusion de l'innovation (75 publications) a démontré que trois de ces cinq caractéristiques influençaient davantage l'adoption d'une innovation ${ }^{26}$. En effet, la compatibilité et les avantages relatifs seraient positivement liés à l'adoption tandis que la complexité y serait négativement liée.

Une étude a confirmé que les caractéristiques qui déterminent l'adoption d'une innovation dans le cas d'environnements informationnels numériques

25. Rogers, 1995, op. cit.

26. Tornatzky, L.G., \& Klein, K.J., 1982, «Innovation Characteristics and Adoption-Implementation : A Meta-analysis of Findings », IEEE Transactions on Engineering Management, EM-29 (1), pp. 28-45. 
sont ceux mentionnés par la théorie de la diffusion de l'innovation de Rogers mais avec quelques modifications ${ }^{27}$. En effet, Moore et Benbasat ont ajouté le concept d'image suivant : l'utilisation d'une innovation améliore le statut social de l'individu. Ces auteurs ont également distingué deux dimensions liées à l'observabilité : la visibilité de l'innovation (visibility) et la possibilité d'en démontrer les résultats (demonstrability). Ils ont par la suite testé leur modèle, en 1995, et ont démontré que le volontarisme, les normes sociales en place et l'ensemble des caractéristiques décrites précédemment étaient les éléments qui influençaient le plus l'adoption.

\section{QUAND ÉMOTION ET COGNITION INTERAGISSENT}

Utilité, utilisabilité et acceptabilité d'un système entretiennent des liens étroits ${ }^{28}$. Pour qu'une bibliothèque numérique soit utilisée, il faut qu'elle soit d'une part, utile et d'autre part, utilisable. L'utilité suggère que le système répond à un besoin clairement identifié et à des attentes. L'utilisabilité correspond au "degré selon lequel un produit peut être utilisé, par des utilisateurs identifiés, pour atteindre des buts définis avec efficacité, efficience et satisfaction, dans un contexte d'utilisation spécifié » (norme ISO 9241-11,1998). Utilité et utilisabilité peuvent être représentés par deux axes orthogonaux, indépendants : en effet, quel que soit le système considéré, nous pouvons trouver des exemples de dispositifs très utiles mais inutilisables (car trop complexes par exemple) ou au contraire très aisément utilisables mais peu utiles. Mais, l'acceptation et l'utilisation d'un système n'obéissent pas à une règle mathématique du type «si..., alors... ». Certains paramètres plus subjectifs sont à prendre en compte tels que, les émotions et de manière générale, l'affect. Paradoxalement, ce n'est que depuis très récemment que les chercheurs en psychologie spécialistes des comportements des usagers interagissant avec des environnements numériques se sont intéressés aux affects et à leurs impacts sur les comportements. Or, il est évident que la motivation, le plaisir à utiliser un système et la satisfaction jouent un rôle prépondérant dans les usages. Très concrètement, un système peut être d'une part, utile, d'autre part, utilisable et néanmoins, ne pas satisfaire l'usager. Cette non-satisfaction se traduit généralement par un abandon du système à court ou moyen terme. De plus, nous pouvons constater un écart considérable entre les environnements numériques plébiscités par les usagers (notamment les jeunes) et la sobriété des interfaces des bibliothèques numériques existant actuellement. Trop souvent, le plaisir associé à l'utilisation est mis de côté dans les produits « sérieux».

À l'aide d'interviews menées auprès de quatorze chercheurs et enseignantschercheurs universitaires en sciences humaines (département d'histoire) et en

27. Moore, G.C., \& Benbasat, I., 1991, « Development of an Instrument to Measure the Perceptions of Adopting an Information Technology Innovation ", Information Systems Research, 2, pp. 192-222.

28. Tricot, A., Plégat-Soutjis, F., Camps, J.-F., Amiel, A., Lutz, G., \& Morcillo, A., 2003, « Utilité, utilisabilité, acceptabilité: interpréter les relations entre trois dimensions de l'évaluation des EIAH ». Actes de la conference EIAH, 15-17 avril ATIEF, ULP et INRP, Strasbourg, pp. 391-402. 
lettres (département d'anglais), Rimmer et al. ${ }^{29}$ ont cherché à comprendre pourquoi ces usagers n'utilisaient pas les bibliothèques numériques alors que celles-ci étaient à l'évidence utiles et utilisables. Ces auteurs se sont alors intéressés à la façon dont ces usagers se représentaient les bibliothèques numériques, en comparant ces représentations avec celles que ces mêmes usagers avaient quant aux bibliothèques " traditionnelles ». S'ils se sont intéressés à ces utilisateurs (chercheurs et enseignants-chercheurs en histoire et en anglais), c'est surtout parce qu'une étude antérieure avait montré que ces derniers faisaient partie des plus réticents pour utiliser les environnements numériques documentaires à leur disposition. Ils ont pu identifier plusieurs obstacles à l'utilisation des bibliothèques numériques pour ces usagers :

- les chercheurs et enseignants-chercheurs regrettent l'uniformité des présentations dans les bibliothèques numériques. En effet, les objets « livres » et " documents » revêtent une importance capitale pour ces spécialistes en histoire et en lettres. L'un des plaisirs de ces experts est de manipuler et d'être au contact de ces objets très différents les uns des autres. Or, en dématérialisant les documents, la numérisation confère un aspect jugé « froid » et « distant»;

- lorsqu'ils utilisent une bibliothèque numérique, ces mêmes experts n'ont plus l'impression d'appartenir à une communauté d'utilisateurs et/ou de spécialistes d'un domaine spécifique. En effet, un autre de leur plaisir évoqué est celui de se retrouver, de se croiser et d'échanger dans les rayonnages des bibliothèques « traditionnelles ». En d'autres termes, dans une bibliothèque numérique, ces experts ne perçoivent plus l'espace social qui leur est cher ;

- liés aux deux points précédemment cités, les chercheurs et enseignantschercheurs en histoire et en lettres déclarent ne pas retrouver les émotions qu'ils ont en travaillant dans une bibliothèque traditionnelle : plaisir de butiner, de feuilleter les ouvrages, d'échanger avec un collègue ou un étudiant croisé au détour d'un rayonnage, etc. ;

- même si de nombreuses études ont montré les spécificités et efficacités des stratégies de recherche d'information des experts d'un domaine dans les environnements numériques ${ }^{30}$, le butinage reste une façon de rechercher des informations couramment utilisée par les experts. Or, le butinage est plus difficile à réaliser dans une bibliothèque numérique ;

- enfin, l'un des arguments scientifiques avancés par les experts interrogés pour expliquer leur très faible utilisation des bibliothèques numériques est

29. Rimmer, R.J., Warwick, C., Blandford, A., Gow, J., \& Buchanan, G., 2008, « An examination of the physical and the digital qualities of humanities research », Information Processing and Management, 44, pp. 1374-1392.

30. Dinet, J., \& Rouet, J.-F., 2002, « La recherche d'information : processus cognitifs, facteurs de difficultés et dimension de l'expertise ", in C. Paganelli (Ed.), Interaction homme-machine et recherche d'information, Hermès, Paris, pp. 133-161 ; Marchionini, G., 1995, Information Seeking In Electronic Environment, Cambridge University Press, Cambridge, England. 
qu'ils disent éprouver énormément de difficultés pour apprécier et juger la valeur historique ou littéraire des œuvres et documents lorsque ces derniers sont "dématérialisés ». La dimension physique (au sens de sensorialité) semble donc être une caractéristique importante pour les experts interrogés, cette dimension étant perdue dans les environnements numériques.

Dans une autre étude récente s'étant intéressée à cette interaction entre les comportements réels des usagers et leurs émotions (ou affects) ${ }^{31}$, les auteurs ont analysé les comportements de 41 usagers, tous universitaires (chercheurs, documentalistes, doctorants), lors de scenarii prédéfinis de recherches documentaires dans la base de données ScienceDirect. L'une des originalités de ce travail est que plusieurs types d'indicateurs ont été recueillis, afin d'obtenir des données quantitatives et qualitatives. Ces indicateurs ont pu être obtenus grâce à l'emploi de plusieurs techniques en parallèle : 1 . les verbalisations concomitantes : durant la réalisation de leurs recherches documentaires, les usagers étaient invités à exprimer et décrire leurs propres comportements ; 2. l'enregistrement audio-vidéo des usagers : durant tout le déroulement des séances de recherche documentaire, chaque usager était filmé par une caméra placée en trois-quarts avant. Ainsi, les expressions faciales et éventuelles "décrochages » de l'utilisateur pouvaient être capturés ; 3 . le traçage des parcours : toutes les actions des usagers sur l'écran étaient enregistrées (requêtes produites, déplacements de la souris, etc.).

Trois indicateurs ont particulièrement attiré l'attention des auteurs : le temps de chacune des phases de recherche documentaire (session length), une phase débutant lors de la production d'une requête et se terminant lorsqu'une nouvelle requête est produite ; le rythme (pace) des phases de recherche documentaire, correspondant au ratio calculé en divisant le nombre de pages consultées par le temps de la session considérée ; les pauses (pauses), c'est-à-dire les moments durant lesquels l'usager ne réalise aucune action mais traite les documents (au sens de lecture-compréhension). Était considéré comme une pause tout moment séparant deux actions, ce moment devant être égal à au moins deux fois le temps moyen d'une action.

Si de nombreux biais méthodologiques empêchent toute généralisation des résultats obtenus par les auteurs dans cette étude, plusieurs remarques sont importantes à relever :

- les temps des sessions des recherches documentaires sont relativement courts (en moyenne, 25 minutes). En d'autres termes, en moyenne, un usager expert modifie sa recherche toutes les 25 minutes lorsqu'il utilise ScienceDirect. Or, la lecture et la compréhension d'un article scientifique contenu dans cette base de données nécessite, a priori, un temps supérieur. Nous pouvons donc émettre l'hypothèse que les usagers se contentent d'un traitement superficiel des documents. L'utilisation de compétences métatextuelles possédées par

31. Tenopir, C., Wang, P., Zhang, Y., Simmons, B., \& Pollard, R., 2008, « Academic users' interactions with ScienceDirect in search tasks : Affective and cognitive behaviors ", Information Processing and Management, 44, pp. 105-121. 
ces usagers experts peut expliquer en partie cet apparent " survol » des documents : en effet, ces utilisateurs experts savent où et comment prélever les informations pertinentes dans les articles scientifiques de manière rapide et efficace (par exemple, les premières phrases des paragraphes, les titres et sous-titres) et savent ne pas prêter attention à d'autres informations moins importantes (par exemple, certains détails méthodologiques);

- les usagers parviennent sans difficulté à exprimer leurs émotions et sentiments durant leurs activités de recherche documentaire. Les émotions et affects positifs (par exemple, « je suis certain que le système va trouver », «j'ai toujours été satisfait avec ce système ») sont significativement plus nombreux que les émotions et affects négatifs (par exemple : " c'est trop vague ", "le système est trop lent »), exprimant ainsi la satisfaction des usagers à l'égard du système ;

- lors d'une phase de recherche documentaire, le nombre moyen de pauses est de 4. Très logiquement, les pauses correspondent en majorité d'une part, aux moments où les listes des articles proposés par le système sont affichées et d'autre part, aux moments où les articles dans leur intégralité sont affichés. En d'autres termes, les pauses correspondent à des temps de lecture, d'évaluation et de sélection des informations. D'autres pauses plus courtes correspondent à des temps durant lesquels les usagers disent réfléchir à une nouvelle requête documentaire et/ou un changement de stratégie de recherche (affiner la recherche avec des termes spécifiques ? élargir avec des termes génériques? etc.).

Quoi qu'il en soit, l'intérêt de cette étude est double : d'une part, montrer la complémentarité de différentes techniques de recueil de données comportementales en associant des techniques telles que les verbalisations concomitantes et les enregistrements audio-vidéos ; d'autre part, montrer qu'il est important d'associer les données quantitatives (performances des usagers, temps pour réaliser l'activité, etc.) à des données qualitatives (impression ressenties, émotions, etc.).

\section{Perspectives}

Indéniablement, les nouvelles technologies et les environnements numériques facilitent notre existence notamment en matière de recherche d'information et de recherche documentaire. Mais, si les bénéfices sont évidents, la facilité d'accès à ces nouveaux environnements a tout de même bouleversé nos comportements et ce, à plusieurs niveaux ${ }^{32}$ :

- spatio-temporel : aujourd'hui, l'usager n'est plus tributaire des horaires d'une bibliothèque puisqu'il peut interroger des bases et banques de données à toute heure de la journée et de la nuit. De même, il est aujourd'hui possible d'interroger et de rechercher des informations depuis des milliers

32. Dinet, 2008, op. cit.; Marchionini, 1995, op. cit. 
de kilomètres, sans bouger de chez soi pourvu que l'on possède les outils adéquats minimums (un ordinateur, une connexion Internet, etc.) ;

- comportemental : les supports, sources, natures et types d'informations auxquels peut accéder tout utilisateur sont désormais en augmentation constante et très diverse. Les comportements se sont donc considérablement modifiés. De plus, la recherche d'information était auparavant du seul ressort d'experts (des bibliothécaires, des documentalistes, etc.) auxquels l'usager devait généralement s'adresser ;

- cognitif : puisque les (res)sources d'informations ne cessent de se multiplier et de se diversifier, l'usager a dû s'adapter et surtout modifier ses stratégies. Parfois, il lui arrive de se perdre dans les méandres hypertextuels ; souvent, il lui arrive de ne pas trouver ce qu'il cherche;

- économique : que ce soit au niveau des individus ou des sociétés et entreprises, la recherche d'information représente un enjeu très important. Aussi, les investissements des particuliers et des entreprises en relation avec la recherche d'information ne cessent d'augmenter ;

- sociétal : à un niveau plus macroscopique, les enjeux associés à la recherche d'information ne sont pas moins importants. Par exemple, l'explosion des (res)sources d'informations rendent difficiles certaines tâches qu'il était jusqu'alors relativement aisé de réaliser (par exemple, l'identification de la source et donc de la crédibilité des informations). De plus, même si quelques efforts sont réalisés en faveur des publics spécifiques (e.g., jeunes enfants, seniors, publics empêchés, personnes handicapées), il est évident que les nouveaux environnements numériques sont pour la plupart inaccessibles pour ces publics.

S’il est vrai que les nouvelles technologies sont censées être au service de l'être humain, force est de constater qu'elles posent un grand nombre d'interrogations et créent souvent des obstacles à la réalisation de nos activités, qu'elles soient quotidiennes ou plus rares. À cela s'ajoutent les «fausses croyances » liées aux nouvelles technologies. Il est donc urgent de poursuivre les études s’intéressant aux comportements réels et facteurs de difficultés des usagers.

\section{JÉRÔME DINET}

